\title{
Nobiletin Reduces Lipid Accumulation in Sebocytes and Inhibits PPAR Delta Activation in Epidermal Tissue Models
}

\author{
Elena Fedorova ${ }^{1}$, Shiming Li $^{2}$, G Luca Gusella ${ }^{1}$, and Arevik Mosoian ${ }^{1, *}$ \\ ${ }^{1}$ SkinAxis, 140 East Hanover Avenue, Cedar Knolls, New Jersey, USA \\ ${ }^{2}$ Beijing University of Chemical Technologies, Beijing, China
}

*Corresponding author: Arevik Mosoian, SkinAxis, 140 East Hanover Avenue, Cedar Knolls, New Jersey, USA, Tel: 347-707-0829; E-mail: Arevik.mosoian@skinaxis.com

Received: 23 Jul, 2021 | Accepted: 31 Aug, 2021 | Published: 10 Sep, 2021

Citation: Fedorova E, Li S, Gusella GL, Mosoian A (2021) Nobiletin Reduces Lipid Accumulation in Sebocytes and Inhibits PPAR Delta Activation in Epidermal Tissue Models. J Clin Lab Med 6(1): dx.doi.org/10.16966/2572-9578.139

Copyright: (C) 2021 Fedorova E, et al. This is an open-access article distributed under the terms of the Creative Commons Attribution License, which permits unrestricted use, distribution, and reproduction in any medium, provided the original author and source are credited.

\begin{abstract}
Background: Acne vulgaris is a chronic inflammatory disease that affects the majority of the population at some point in their lifetime. Acne pathogenesis is multifactorial with four primary contributors that play a pivotal role in the formation of acne lesions: inflammation, androgeninduced sebum production, abnormal keratinization, and bacterial colonization. Recent studies have demonstrated the anti-inflammatory, anticarcinogenic, anti-diabetic, and anti-lipid properties of certain Polymethoxylated Flavones (PMF) derivatives.
\end{abstract}

Objective: To study the anti-inflammatory activity and inhibition of lipid production by nobiletin and a mixture of PMF in three-dimensional (3D) epidermal models and sebocytes.

Methods: Oil Red O staining of primary human sebocytes treated with nobiletin and Mixture PMF was used to test the Inhibition of lipid production. Microarray analysesin three-dimensional (3D) epidermal tissue models were used. QuantiSig ${ }^{\circledast}$ luciferase reporter assay was used to study the effect of nobiletin and Mixture PMF on activation of nuclear receptor Peroxisome Proliferator-Activated Receptor (PPAR) $(\alpha, \beta / \delta, \gamma)$.

Results: Nobiletin and Mixture PMF significantly reduced sebum production in primary human sebocytes and inhibited PPAR activation. The known anti-acne compound retinoic acid (RA) stimulated PPAR activation, suggesting possible different sebum reduction pathways. Nobiletin and Mixture PMF down-regulated PPAR gene expression and inhibited cytokines and immune receptors involved in the inflammatory response. Nobiletin downregulated cytochromes P450 cluster of CYP family of genes: CYP4F22, CYP4B1, CYP4Z2P suggests inhibition of lipid production by nobiletin via reduction of hormonal stimulation.

Conclusions: Nobiletin and Mixture PMF inhibited the inflammatory cascade, androgenic pathway in 3D human epidermal tissue models compared to the vehicle-treated cells. Nobiletin treatment of epidermal tissues reduces lipid accumulation in primary human sebocytes, inhibits PPAR $\delta$ activation compared to the vehicle-treated cells.

Keywords: Polymethoxylated flavones; Microarray analyses; Cytochromes P450

Abbreviations: PPAR: Peroxisome Proliferator-Activated Receptor $(\alpha, \beta / \delta, \gamma)$; Mixture PMF: Polymethoxylated Flavones Mixture; NF-kB: NFkappaB; MMP: Matrix Metalloproteinase; RA: Retinoic Acid

\section{Introduction}

Acne is the most common skin condition in the United States of America, affecting $80 \%$ of people between 11 and 30 years of age $[1,2]$. It equally affects genders, skin types. By age $21,90 \%$ of men and $80 \%$ of women will have acne vulgaris [3]. Acne can persist in affected patients throughout adulthood, or appear de novo at any time and does not substantially decrease until after age $44(\mathrm{P}<0.001)$ [1]. The acne treatment field is witnessing a shift from formulated drugs limited to reducing sebum production to compounds targeting specific pathways of the immune system, which could be administered safely and available over the counter.
Acne vulgaris is a chronic inflammatory disease: factors in these conditions are the increased sebum production, hypercornification, and abnormal differentiation of keratinocytes of the upper part of the follicle. Hormones are other contributing factors that influence the severity of acne. Additionally, skin resident bacteria play a role in disease pathogenesis. The main three families of bacteria on the skin surface belong to Corynebacteria, Propionibacteria, and Staphylococci [4]. The interplay between members of this cutaneous microbiota and innate immunity is essential for the maintenance of healthy skin. Thus acne pathogenesis is multi factorial with four primary factors that play a pivotal role in the formation of acne lesions: inflammation, androgen-induced sebum production, abnormal keratinization, and 
bacterial colonization of Cutibacterium acnes in the pilosebaceous unit [5].

Recent evidence suggests inflammation as the inciting factor rather than activation of sebaceous glands, with proof of significant inflammatory factors surrounding the pilosebaceous unit even in clinically normal skin units in acne patients [6]. A new understanding of how Cutibacterium acnes (C. acnes) induce the inflammatory cascade has provided a new paradigm for the management of acne. Recognition of microbes, such as C. acnes, by the innate immune system is the body's first line of defense against Pathogen-Associated Molecular Patterns (PAMPs) and Damage-Associated Molecular Patterns (DAMPs). C. acnes triggers innate immune response via the activation of toll-like receptors 2 and 6 (TLR2/6) and importantly the inflammasome [7]. The inflammasome, a caspase 1-activating cytoplasmic complex induces the secretion of crucial proinflammatory cytokines, including tumor necrosis factor $\alpha$ (TNF- $\alpha$ ), IL-1, IL-6, IL-8, and IL-12 [8].

It is reported that nobiletin modulates a wide array of signalling pathways involved in the immune response, supporting its potential for use against inflammatory diseases such as acne. In contrast to RA, the main anti-acne treatment, PMFs derived from citrus peels prevented UVB-induced photo-inflammation by suppressing the expression of COX-2 in human keratinocytes [3,9]. Recent studies have demonstrated the anti-inflammatory, anti-carcinogenic, antidiabetic, and anti-lipid properties of certain PMF derivatives [10-13].

Our data show that nobiletin treatment of epidermal tissues or sebocytes inhibits a) the inflammatory cascade; b) androgenic and estrogenic pathways; c) lipid production and d) PPAR $\delta$ activation.

\section{Materials and Methods}

\section{Reagents}

Nobiletin (N; Deep Sense LLC, USA) stock solution was prepared in DMSO and tested at $0.02 \mathrm{mg} / \mathrm{mL}$ final concentration. 5-Demethylnobiletin (5-D-Nob; Deep Sense LLC, USA) stock solution was prepared in DMSO and tested at $0.01 \mathrm{mg} / \mathrm{mL}$ and $0.005 \mathrm{mg} / \mathrm{mL}$ final concentrations. Rosiglitazone (Rgz; Cayman Chemical, MI, USA) stock solution was prepared in DMSO and was used at concentration $9 \mu \mathrm{g} / \mathrm{ml}$ as a positive control for PPAR activation. Retinoic acid (RA; Sigma, MO, USA) stock solution was prepared in DMSO and tested at $1 \mu \mathrm{M}$ final concentration in cell culture medium.

\section{Cell culture}

Primary human epidermal keratinocytes derived from the foreskin of a single donor were purchased from PromoCell (Heidelberg, Germany) and grown in supplemented Epilife medium (Thermo Fisher Scientific, NJ, USA). Differentiated 3D epidermal tissues were cultured on tissue inserts (Thermo Fisher Scientific/Nunc, CA, USA) at SkinAxis, LLC (Cedar Knolls, NJ, USA). The cells were submerged in supplemented Epilife growth medium for 48 hours and then grown in keratinocyte defined medium (KDM, SkinAxis, NJ, USA) at the air-liquid interface for 8 days. Keratinocytes and cultured epidermal tissues were maintained at $37^{\circ} \mathrm{C}$ with $5 \% \mathrm{CO}_{2}$ and $\sim 95 \%$ humidity.

$3 \mathrm{D}$ epidermal tissues were topically treated with $25 \mu \mathrm{l}$ of compounds, positive or negative controls for 24 hours. After incubation, tissues were washed in PBS (Thermo Fisher Scientific), and total RNA was extracted using a Qiagen RNeasy kit (Qiagen, CA, USA) according to manufacturer's instructions.

\section{Cytotoxicity assay}

Cytotoxicity was evaluated using the Cell Titer 96 Aqueous One (Promega, WI, USA) basic test according to the manufacturer's instructions. Keratinocytes were seeded in a 96-well plate, cultured overnight, and then incubated for 24 hours in the presence of the compounds. Untreated and 5\% DMSO-treated cells were used as a negative and positive control, respectively. All treatments were performed in triplicate. Treatments with compounds resulting in the inhibition of viability of more than $20 \%$ of the negative control values were considered cytotoxic.

\section{Testing expression of PPAR promoter with luciferase reporter in response to nobiletin treatment}

Cultured primary human epidermal keratinocytes were infected with VSV-G envelope pseudotyped lentivector carrying the luciferase reporter gene under the control of the PPAR, $\beta / \delta, \gamma$ ) promoter (SkinAxis QuantiSig ${ }^{\circledR}$ system; SkinAxis, NJ, USA). Transduced keratinocytes were maintained at $37^{\circ} \mathrm{C}$ with $5 \% \mathrm{CO}_{2}$ and $95 \%$ humidity for 24 hours. Treatment with different concentrations of compounds was done for 24 hours followed by luciferase and protein concentration assays.

\section{Treatments of cultured epidermal tissues}

Epidermal tissues (four biological replicates for each condition) cultured in tissue inserts (Thermo Fisher Scientific/Nunc) were topically treated with $25 \mu \mathrm{l}$ of compounds, positive or negative controls and addition of the treatments to cell culture medium. After 24-hour incubation, tissues were washed in PBS (Thermo Fisher Scientific), and total RNA was extracted using a Qiagen RNeasy kit (Qiagen, CA, USA) according to the manufacturer's instructions. RNA concentration and purity were determined using a Nano drop IMPLEN spectrophotometer.

\section{RNA quality control and microarray analysis}

The integrity of total RNA samples and RNA quality was confirmed by Advanced BioMedical Laboratories (Cinnaminson, NJ, USA). A proprietary algorithm that takes several QC parameters into account (e.g. $28 \mathrm{~S} / 18 \mathrm{~S}$ peak area ratios, unexpected peaks in the $5 \mathrm{~S}$ region, etc.) was used to calculate the RNA Integrity Numbers (RIN). A RIN number of 10 indicated perfect RNA quality; a RIN number of 1 indicates degraded RNA. RNA with a RIN number $\geq 8$ is of sufficient quality for gene expression profiling experiments. The RIN number for all RNA samples was $>8$. Microarray analysis was performed using Affymetrix Human Clariom D array processing by Advanced BioMedical Laboratories (Cinnaminson).

\section{Statistical analyses}

The differential gene expression was obtained using a threshold of 0.05 for statistical significance ( $p$-value) and a log fold change of expression with an absolute value of at least 0.6. The microarray data herein presented reached statistically significant nominal $\mathrm{p}$ values. Gene expression was further analyzed using Differential Expression Analysis and Pathway Analysis (iPathway Guide; [14] by Advaita Bioinformatics Services (Plymouth, MI) in the context of pathways obtained from the Kyoto Encyclopedia of Genes and Genomes (KEGG) database (Release 84.0+/10-26, Oct 17) [15], gene ontologies from the Gene Ontology Consortium database (2017-Nov 6) [16], miRNAs from the miRBase (Release 21) and TARGETSCAN (Targets can version: Mouse:7.1, Human:7.1) databases [17,18] and diseases from the KEGG database (Release 84.0+/10-26, Oct 17) [19].

Data are presented as mean \pm SD. Two-tailed t-test or two-way ANOVA with Bonferroni post-test were used for statistical analyses 
using Graph Pad Prism software. P value $<0.05$ was considered statistically significant.

\section{Results}

Evaluation of intracellular lipids in primary human sebocytes treated with nobiletin or Mixture PMF

Preliminary experiments were carried out to determine the noncytotoxic range of nobiletin and a mixture of Nobiletin and Tangeritin (Mixture PMF) which has solubility and permeation similar to Nobiletin (data not shown). Normal human sebocytes (ZenBio, Research Triangle Park, NC) were cultured in the presence of noncytotoxic concentration $(10 \mu \mathrm{g} / \mathrm{ml})$ of nobiletin or Mixture PMF for 72 hours before their effect was assessed by measuring the intracellular lipids content following Oil Red O staining (Figure 1A). Oil Red O staining was quantified by using Image $J$ for the percentage of area stained positively for Oil Red O. Quantitative analysis revealed that the percentage of area stained positively for Oil Red $\mathrm{O}$ was significantly reduced in treated cells compared to cells cultured in growth medium (Figure 1B).

\section{Inhibition of PPARs by nobiletin and Mixture PMF}

Nobiletin and Mixture PMF cytotoxicity curves were first determined in primary human epidermal keratinocytes derived from the foreskin of a single donor (PromoCell, Heidelberg, Germany) and grown in EpiLife supplemented medium (Thermo Fisher, NJ, USA). The nuclear receptor peroxisome proliferator-activated receptor gamma (PPAR $\gamma$ ) controls the expression of different genes involved in the regulation of lipid pathways.

Studies in human sebocytes have raised the possibility that PPAR $\gamma$ modulation can lead to alteration of lipid production and may be useful in the treatment of acne [20]. The effect of nobiletin and Mixture PMF on the PPAR pathway was assessed using SkinAxis' QuantiSig ${ }^{\oplus}$ reporter system in which primary human keratinocytes are engineered with a luciferase gene under the control of the PPAR $(\alpha, \beta / \delta, \gamma)$ promoter.
Nobiletin showed a decrease of reporter expression as compared to control vehicle-treated keratinocytes by $58 \%$ and $63 \%$ in the presence of PPAR activator -roziglitazone respectively indicating significant suppression of the PPARs activation (Figure 2). The mixture of PMFs at a final concentration of $10 \mu \mathrm{g} / \mathrm{ml}$ lowered the reporter expression by $49 \%$ and $51 \%$ in the presence of rosiglitazone compared to control treated cells (Figure 2). As expected, significant stimulation of the reporter was observed with the positive control Rosiglitazone (151\% Figure 2). RA at $1 \mu \mathrm{M}$ produced a significant stimulation (214\%) of the reporter expression compared to the vehicle control (Figure 2). Our data on RA stimulation of the PPAR pathway are in agreement with other studies showing activation of PPAR by RA $[21,22]$.

\section{Microarray analyses of 3D human epidermal tissues treated with nobiletin}

To evaluate the effect of Nobiletin $(25 \mu \mathrm{M})$, Mixture PMF $(10 \mu \mathrm{g} /$ $\mathrm{ml})$, and $\mathrm{RA}(1 \mu \mathrm{M})$ on gene expression profile in cultured human epidermal tissues (Figure 3 ) microarray analyses was used. Nobiletin and RA treatment affected the PPAR pathway by triggering different gene expression profiles. Microarray results confirmed our QuantiSig ${ }^{\bullet}$ data showing RA stimulation but Nobiletin and Mixture PMF downregulation of genes involved in the PPAR pathway. Moreover, the PPAR $\delta$ gene was significantly down-regulated by Nobiletin and Mixture PMF treatment ( -0.731 and $-1.368 \log \mathrm{Fc}$ ) respectively), but was not affected by RA treatment. These data suggest that Nobiletin may trigger different sebum reduction pathways than RA.

Sato et al. 2007 showed that a mechanism of suppression of sebum production by Nobiletin includes inhibition of Diacylglycerol Acyltransferase (DGAT) [23]. Our microarray data confirmed the reduction of the DGAT gene expression not only by Nobiletin $(-1.241)$ and Mixture PMF (-1.417), but also by RA (-1.1), suggesting that the inhibition of DGAT gene expression is common to Nobiletin, Mixture PMF as well as RA. Importantly, we also showed that Nobiletin downregulated the expression of cytokines and immune receptors involved in inflammation (Table 1).

Cytochrome P450 (CYPs) regulate skin homeostasis and metabolic
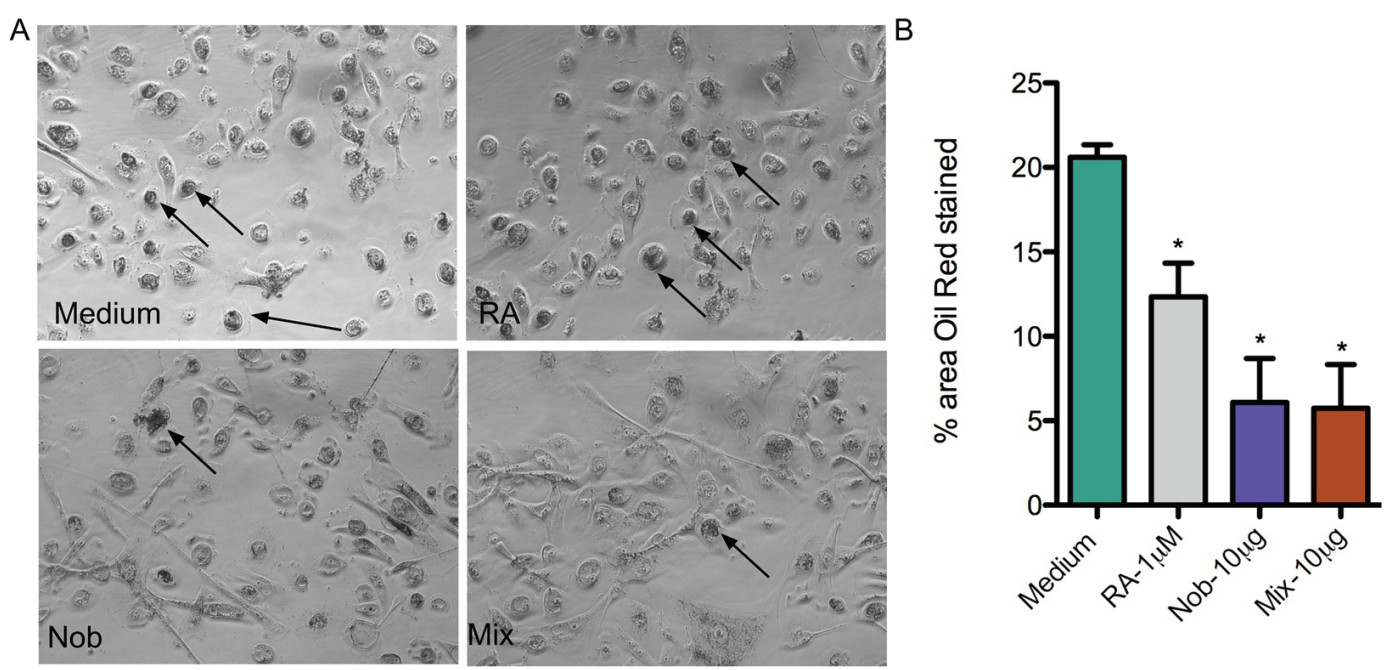

Figure 1A, 1B: Reduction of Oil Red O staining in primary human sebocytes treated with nobiletin and mixture of PMF. 1A) Microscopy detection of lipid droplets in sebocytes treated for 72 hours with either with medium control, $1 \mu \mathrm{M}$ retinoic acid (RA) positive control, $25 \mu \mathrm{M}$ Nobiletin, or $10 \mu \mathrm{g} /$ $\mathrm{ml}$ Mixture PMF (Mix). Arrows indicate lipid droplets in cells. Original magnification: 200X. 1B) Quantitative analysis using Image revealed that the percentage of area stained positively for Oil Red was significantly lower in treated cells than in growth medium cultured cells. One-way ANOVA: The data are expressed as mean $\pm S D, n=6, * P<0.05$ vs. growth medium treated cells. 


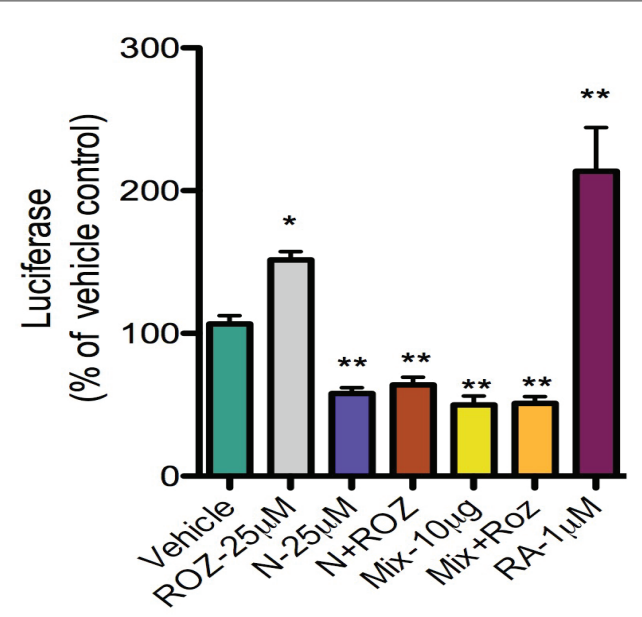

Figure 2: Nobiletin and Mixture PMF reduce PPARs activation. Activation of the PPAR promoter in cultured human keratinocytes treated as indicated for 24 hours. (The PPAR pathway activator Rosiglitazone (RGZ) at $25 \mu \mathrm{M}$ was used as positive control. The known anti-acne compound, Retinoic acid (RA) at concentration $1 \mu \mathrm{M}$, was used as a reference, nobiletin (at concentration $25 \mu \mathrm{M}$ ) and mixture Polymethoxyflavones (PMF) at $10 \mathrm{ug} / \mathrm{ml}$. All readings were normalized to total protein concentration and data are reported relatively to the expression of the reporter in the vehicle control (Vehicle negative control: $100 \%)$. One way ANOVA: ${ }^{*} P<0.05 ;{ }^{* *} P<0.01$.

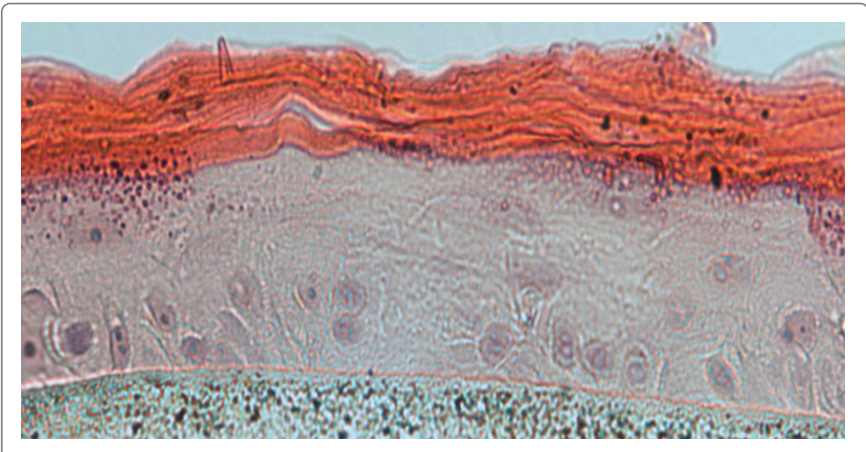

Figure 3: Hematoxylin and eosin staining of three-dimensional epidermal tissue.

barrier of the skin through activation or inactivation of different pathways. Cytochrome $\mathrm{P} 450$ proteins catalyze many reactions involved in drug metabolism, including the synthesis of cholesterol, steroids, and other lipids [24].

The local production of androgens and estrogens from steroid precursors is initiated by locally expressed CYPs [25].

Our microarray studies revealed that Nobiletin and Mixture PMF down-regulated a cluster of CYP genes in the cytochrome P450 family: CYP1A1, CYP4F22, CYP2S1, CYP1B1, CYP34, CYP39A1 (Table 2) suggesting that Nobuletin could decrease lipid synthesis. Local production of androgens and estrogens in the skin is regulated by a combination of CYPs (CYP11A1, CYP11B1, CYP17A1) and steroid dehydrogenases. Down-regulation of indicated CYPs by Nobiletin treatment was not statistically significant. However, nobiletin treatment of epidermal tissues down-regulated the estrogen signaling pathway by reducing the expression of genes involved in this pathway (Table 3).
Table 1: Treatment of epidermal tissues with nobiletin down regulated cytokines, receptors and nuclear factor involved in inflammatory response.

\begin{tabular}{|l|c|c|}
\hline \multicolumn{1}{|c|}{ Gene Symbol } & Log Fc & p-value \\
\hline IL36RN & -2.567 & $1.000 \mathrm{e}-6$ \\
\hline IL7R & -1.618 & $2.197 \mathrm{e}-6$ \\
\hline IL1RN & -1.618 & $1.056 \mathrm{e}-4$ \\
\hline IL1R2 & -1.604 & $5.065 \mathrm{e}-4$ \\
\hline IL22RA1 & -0.0 .796 & $7.745 \mathrm{e}-4$ \\
\hline IL20RA & -0.795 & 0.002 \\
\hline IL37 & -0.882 & 0.004 \\
\hline IL36G & -0.664 & 0.004 \\
\hline PPIL6 & 0.795 & 0.005 \\
\hline NF-KB & -0.629 & 0.006 \\
\hline
\end{tabular}

Table 2: Nobiletin treatment of epidermal tissue down-regulated Cytochrome P450 subfamily gene members.

\begin{tabular}{|l|c|c|}
\hline \multicolumn{1}{|c|}{ Gene Symbol } & Log Fc & p-value \\
\hline CYP1A1 & -1.679 & $1.619 \mathrm{e}-6$ \\
\hline Cyp4F22 & -2.331 & $1.000 \mathrm{e}-5$ \\
\hline CYP2S1 & -0.718 & $8.368 \mathrm{e}-4$ \\
\hline CYP1B1 & -1.618 & $1.056 \mathrm{e}-4$ \\
\hline CYP3A4 & -0.655 & 0.011 \\
\hline CYP39A1 & -0706 & 0.001 \\
\hline
\end{tabular}

Table 3: Nobiletin treatment of epidermal tissue down-regulated genes involved in estrogen signalling pathway.

\begin{tabular}{|l|c|c|}
\hline \multicolumn{1}{|c|}{ Gene Symbol } & Log Fc & p-value \\
\hline KRT10 & -2.743 & $3.837 \mathrm{e}-6$ \\
\hline KRT23 & -1.822 & $3.57 \mathrm{e}-6$ \\
\hline MMP9 & -1.113 & $4.618 \mathrm{e}-4$ \\
\hline HBEGF & 0.783 & $1.359 \mathrm{e}-4$ \\
\hline MAPK3 & -1.028 & $1.548 \mathrm{e}-4$ \\
\hline MMP2 & -0.922 & $8.509 \mathrm{e}-4$ \\
\hline
\end{tabular}

\section{Discussion}

Human skin is the largest body organ and is considered as the first line of defense and barrier against infections caused through the skin. Acne vulgaris is a very common inflammatory disease occurring primarily on the face and also rare on the upper arms, trunk, and back, which is caused by different bacterial species [4]. Despite advancement in science, the development of natural herbal treatments for skin disorders becomes an essential contributor for not only managing the side effects of conventional medicines but also more effectively treating skin disease. Herbal extracts and their phyto medicines make a fundamental contribution to human health care.

PMFs are reported to have several biological effects in vivo and in vitro, including antioxidant, anti-inflammatory, and anti-tumorigenic activities [13,26,27]. Our microarray data are in agreement with published reports on the anti-inflammatory activity of nobiletin [28]. We showed that the Nobiletin treatment of epidermal tissues downregulated the expression of cytokines and immune receptors involved 


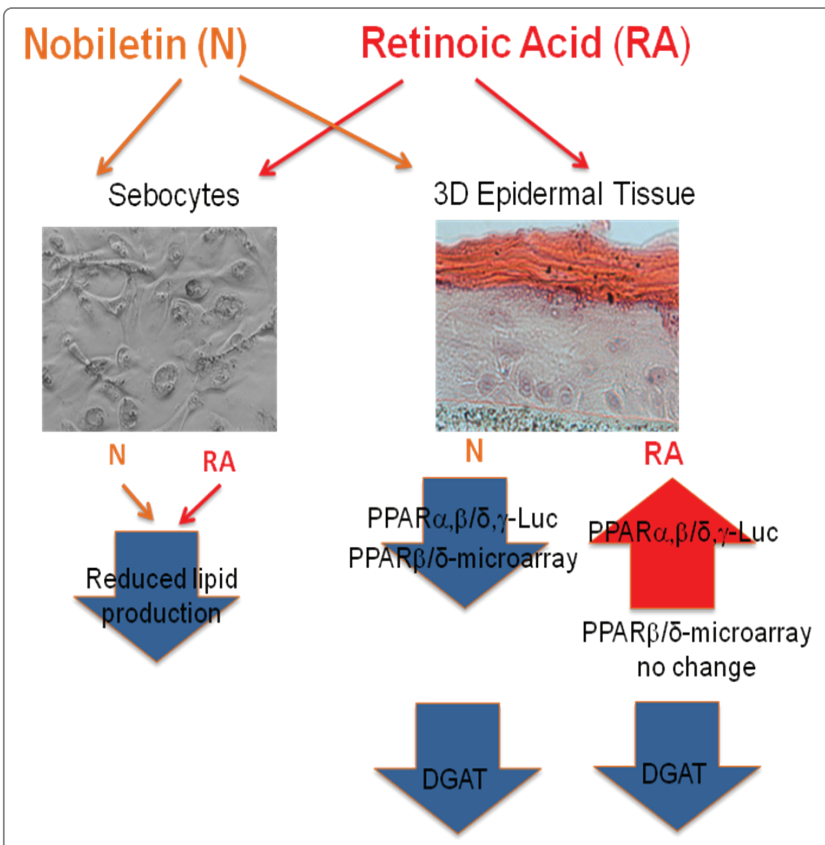

Figure 4: Graphical summary of the work.

in inflammation. NF- $\mathrm{kB}$ activation plays a key role in innate immunity and inflammation, and inhibition of NF- $\mathrm{kB}$ has been considered as a highly promising anti-inflammatory therapy. We found that the nobiletin and mixture PMFs strongly down-regulated NF- $\kappa B$ and IL-36RN gene in addition to other key cytokine genes resulting in lowered IL-RA proteins responsible for causing inflammatory skin diseases such as acne and psoriasis.

In this study, we examined in vitro effects of nobiletin on oil production compared to RAs. In-vitro studies with primary human sebocytes were used to determine the effect of nobiletin and PMF mixture on oil production. Sebocytes, despite being of epithelial origin and expressing the number of hormone receptors, are involved in lipid synthesis and metabolism, a function that is normally found in adipocytes. The sebaceous gland can be considered both as a target for hormones as well as an endocrine organ.

Our results are in agreement with the published report from Sato et al., on inhibition of lipogenesis by nobiletin. One of the mechanisms of suppression of sebum production by Nobiletin reported by Sato et al., was considered inhibition of Diacylglycerol Acyltransferase (DGAT) [23] However, while our microarray data confirmed the reduction of the DGAT gene expression not only by Nobiletin and Mixture PMF but also by RA, suggesting that the inhibition of DGAT gene expression is common to Nobiletin, Mixture PMF as well as RA [23].

We have shown that the Mixture PMF and nobiletin inhibition of lipid production in primary human sebocytes is different from the pathways triggered by RA. We discovered that, unlike RA, the PMFs inhibit the PPAR $\delta$ signaling pathway, while RA-stimulated PPAR expression and has no effect on PPAR $\delta$. The graphical summary of the results is presented in figure 4 .

PPAR $\delta$ regulates a multitude of physiological processes associated with lipid metabolism, inflammation, immune response, and proliferation. The PMFs therefore can help modulate antiinflammatory properties that target keratinocytes in the epidermis and sebocytes in the superficial dermis to effectively treat acne vulgaris. They also open opportunities for treatment of other inflammationmediated skin disorders including psoriasis and melanoma.

The metabolic barrier of the skin is composed of cytochromes P450 (CYPs) that regulate its homeostasis through activation or inactivation of different pathways. The cytochrome $\mathrm{P} 450$ proteins are monooxygenases that catalyze many reactions involved in drug metabolism, synthesis of cholesterol, steroids, and other lipids [24]. The local skin steroidogenic system is composed of locally expressed CYPs involved in the local production of androgens and estrogens from steroid precursors [25]. Our microarray studies revealed that Nobiletin and Mixture PMF down-regulated not only a cluster of CYP genes in the cytochromes P450 family but also genes involved in the estrogen pathway, suggesting that Nobuletin could inhibit local production of androgens and estrogens and thus decrease lipid production possibly via the local inhibition of the hormonal pathway.

\section{Data Availability Statement}

All the data used to support the findings of this study are included within the article.

\section{Funding Information}

This work was supported by the funding from SkinAxis LLC.

\section{Competing Interests}

The author confirms that there are no known conflicts of interest associated with this publication and there has been no significant financial support for this work that could have influenced its outcome.

\section{Ethics Approval and Consent to Participate}

Studies reported in this manuscript do not involve animals or human subjects; human cell lines were obtained from indicated companies with de-identified donor information.

\section{Acknowledgments}

We are grateful to Dr. Jessica Langer for advice and critical reading of the manuscript.

\section{References}

1. Goulden V, Stables GI, Cunliffe WJ (1999) Prevalence of facial acne in adults. J Am Acad Dermatol 41: 577-580.

2. Jacob Cl, Dover JS, Kaminer MS (2001) Acne scarring: a classification system and review of treatment options. J Am Acad Dermatol 45: 109-117.

3. Tanaka S, Sato T, Akimoto N, Yano M, Ito A (2004) Prevention of UVB-induced photoinflammation and photoaging by a polymethoxy flavonoid, nobiletin, in human keratinocytes in vivo and in vitro. Biochem Pharmacol 68: 433-439.

4. Grice EA, Kong HH, Conlan S, Deming CB, Davis J, et al. (2009) Topographical and temporal diversity of the human skin microbiome. Science 324: 1190-1192.

5. Suvanprakorn $P$, Tongyen $T$, Prakhongcheep $\mathrm{O}$, Laoratthaphong $\mathrm{P}$, Chanvorachote P (2019) Establishment of an Anti-acne Vulgaris Evaluation Method Based on TLR2 and TLR4-mediated Interleukin-8 Production. In vivo 33: 1929-1934.

6. O'Neill AM, Gallo RL (2018) Host-microbiome interactions and recent progress into understanding the biology of acne vulgaris. Microbiome 6: 177. 
7. Rosen J, Friedman AJ (2014) Inflammatory acne: new developments in pathogenesis and treatment. Cutis 94: 266-267.

8. Kurokawa I, Danby FW, Ju Q, Wang X, Xiang LF, et al. (2009) New developments in our understanding of acne pathogenesis and treatment. Exp Dermatol 18: 821-832.

9. Lee KJ, Park KH, Hahn JH (2019) Alleviation of Ultraviolet-B RadiationInduced Photoaging by a TNFR Antagonistic Peptide, TNFR2-SKE. Mol Cells 42: 151-160.

10. Lin N, Sato T, Takayama Y, Mimaki Y, Sashida Y, et al. (2003) Nove anti-inflammatory actions of nobiletin, a citrus polymethoxy flavonoid, on human synovial fibroblasts and mouse macrophages. Biochem Pharmacol 65: 2065-2071.

11. Luque-Alcaraz AG, Lizardi J, Goycoolea FM, Valdez MA, Acosta AL, et al. (2012) Characterization and antiproliferative activity of nobiletinloaded chitosan nanoparticles. J Nanomater 100.

12. Minagawa A, Otani Y, Kubota T, Wada N, Furukawa T, et al. (2001) The citrus flavonoid, nobiletin, inhibits peritoneal dissemination of human gastric carcinoma in SCID mice. Jpn J Cancer Res 92: 13221328.

13. Ma W, Feng S, Yao X, Yuan Z, Liu L, et al. (2015) Nobiletin enhances the efficacy of chemotherapeutic agents in ABCB1 overexpression cancer cells. Sci Rep 5: 18789

14. Alexa A, Rahnenführer J, Lengauer T (2006) Improved scoring of functional groups from gene expression data by decorrelating $\mathrm{GO}$ graph structure. Bioinformatics 22: 1600-1607.

15. Kanehisa M (2000) Pathway databases and higher order function. Adv Protein Chem 54: 381-408.

16. Gene Ontology Consortium (2001) Creating the gene ontology resource: design and implementation. Genome Res 11: 1425-1433.

17. Agarwal V, Bell GW, Nam JW, Bartel DP (2015) Predicting effective microRNA target sites in mammalian mRNAs. Elife 4: e05005.

18. Panwar B, Omenn GS, Guan Y (2017) miRmine: a database of human miRNA expression profiles. Bioinformatics 33: 1554-1560.
19. Kanehisa M (2002) The KEGG database. Novartis Found Symp 247: 91-101; discussion 101-103, 119-128, 244-152.

20. Mastrofrancesco A, Ottaviani M, Cardinali G, Flori E, Briganti S, et al. (2017) Pharmacological PPARgamma modulation regulates sebogenesis and inflammation in SZ95 human sebocytes. Biochem Pharmacol 138: 96-106.

21. D’Aniello E, lannotti FA, Falkenberg LG, Martella A, Gentile A, et al. (2019) In Silico Identification and Experimental Validation of (-)-Muqubilin A, a Marine Norterpene Peroxide, as PPAR $\alpha / \gamma-\operatorname{RXR} \alpha$ Agonist and RAR $\alpha$ Positive Allosteric Modulator. Mar Drugs 17: 110.

22. Natesan S, Wrice NL, Christy RJ (2019) Peroxisome proliferator activated receptor- $\alpha$ agonist and all-trans retinoic acid induce epithelial differentiation of subcutaneous adipose-derived stem cells from debrided burn skin. J Cell Biochem 120: 9213-9229.

23. Sato T, Takahashi A, Kojima M, Akimoto N, Yano M, et al. (2007) A citrus polymethoxy flavonoid, nobiletin inhibits sebum production and sebocyte proliferation, and augments sebum excretion in hamsters. J Invest Dermatol 127: 2740-2748.

24. Jarrar YB, Lee SJ (2019) Molecular Functionality of Cytochrome P450 4 (CYP4) Genetic Polymorphisms and Their Clinical Implications. Int J Mol Sci 20: 4274.

25. Slominski AT, Zmijewski MA, Semak I, Zbytek B, Pisarchik A, et al. (2014) Cytochromes p450 and skin cancer: role of local endocrine pathways. Anticancer Agents Med Chem 14: 77-96.

26. Zhang L, Zhang X, Zhang C, Bai X, Zhang J, et al. (2016) Nobiletin promotes antioxidant and anti-inflammatory responses and elicits protection against ischemic stroke in vivo. Brain Res 1636: 130-141.

27. Murakami A, Nakamura Y, Torikai K, Tanaka T, Koshiba T, et al. (2000) Inhibitory effect of citrus nobiletin on phorbol ester-induced skin inflammation, oxidative stress, and tumor promotion in mice. Cancer Res 60: 5059-5066.

28. Lin Z, Wu D, Huang L, Jiang C, Pan T, et al. (2019) Nobiletin Inhibits IL-1 $\beta$-Induced Inflammation in Chondrocytes via Suppression of NFKB Signaling and Attenuates Osteoarthritis in Mice. Front Pharmacol 10: 570 . 